Voix et Images

voixetimages

\title{
De la marge et des marginaux
}

\section{Robert Major}

Volume 18, numéro 3 (54), printemps 1993

Littérature, folie, altérité

URI : https://id.erudit.org/iderudit/201051ar

DOI : https://doi.org/10.7202/201051ar

Aller au sommaire du numéro

Éditeur(s)

Université du Québec à Montréal

ISSN

0318-9201 (imprimé)

1705-933X (numérique)

Découvrir la revue

Citer cet article

Major, R. (1993). De la marge et des marginaux. Voix et Images, 18(3), 580-589.

https://doi.org/10.7202/201051ar

Ce document est protégé par la loi sur le droit d'auteur. L'utilisation des services d'Érudit (y compris la reproduction) est assujettie à sa politique d'utilisation que vous pouvez consulter en ligne.

https://apropos.erudit.org/fr/usagers/politique-dutilisation/ 


\section{Essai}

\section{De la marge et des marginaux}

\section{Robert Major, Université d'Ottawa}

La marge est consubstantielle à l'écrit. Au sens propre, d'abord. Pas de page sans marge, pas de lettres sans bordure, pas d'encre noire sans support et encadrement d'espace blanc. Mais au sens plus large, aussi, la réalité littéraire ne saurait exister sans refoulement constant vers ses franges de ce qui ne saurait s'y intégrer de plein droit. Pas de littérature sans marginalité, qui la précède ou la talonne, la conteste ou la nie, mais toujours, ainsi, la constitue dans son espace et son champ. Il faut des confins, des bornes à la chose littéraire tout autant qu'à l'objet-livre. La marge s'oppose à l'écrit, mais également le constitue; elle enclot le texte et l'identifie, mais s'en coupe du fait même. Cette double et dialectique réalité d'inclusion-exclusion fait en sorte que sont associées à la marge une riche cohorte de significations reliées à la marginalité. Les considérations sur la marge, en somme, ne sont ni futiles ni marginales.

Témoin François Paré et son beau livre sur Les Littératures de l'exiguitét ${ }^{-1}$ "J'écris ce livre face à la mer", nous déclare-t-il dans son incipit. Certes, l'attitude est quelque peu romantique. "C'est la mer du Nord, violente, inépuisable, imprévisible." Nous ne sommes pas à Saint-Malo, mais c'est tout comme. Le spectre de Chateaubriand n'est pas loin. Le spectacle de la mer, comme la contemplation des ruines, provoque, on le sait, les plus essentielles réflexions sur la fragilité des projets humains et la précarité des civilisations et des cultures. Fragilité d'autant plus évidente aux Pays-Bas, où se trouve l'auteur, que ce pays ne tient la mer à distance qu'au moyen d'une étroite bande de dunes sauvages, si précaires et pourtant si résistantes.

Mais ce n'est pas la mer qui importe à François Paré: ce sont les rives. De même, ce n'est pas la posture de l'essayiste qui doit nous retenir, mais plutôt le parcours de sa réflexion. Or, c'est en considérant ces dunes sévèrement protégées par les Hollandais, c'est en rêvant à ces marges sacralisées, c'est sous le signe de cette "métaphore vivante" (p. 5) que François Paré nous convie à une réflexion sur "les petites cultures et leur espace littéraire propre» (p. 6). Cette métaphore de la 
marge, à mon tour, je me l'approprie sans vergogne, pour rendre compte d'abord de son essai remarquable, mais aussi de livres récents sur le phénomène de la marginalité culturelle et littéraire.

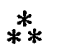

Exiguité. Le mot a été accepté difficilement en français, si l'on en croit Le Robert, qui atteste le terme dès le xIV siècle mais constate son absence totale des dictionnaires des $\mathrm{XVII}^{e}$ et $\mathrm{XVIII}^{e}$ siècles. La fortune du mot est révélatrice du statut de ce qu'il prétend décrire. L'exigu est petit, insuffisant, étroit, modeste, étriqué, minuscule. Médiocre, aussi. Il est refoulé à la marge de ce qui est grand et important. Tel est le statut des petites littératures auxquelles s'intéresse François Paré, dans ce livre qui tient autant de l'essai que du journal intime, de l'analyse critique que du cri du cœur, de l'exposé savant que du plaidoyer.

La facture du livre, d'ailleurs, étonne d'abord le lecteur. L'écriture aussi est exiguë. Se succèdent, chacune avec son titre propre, des séquences de longueurs inégales, quelquefois un paragraphe ou une demi-page, le plus souvent une page, rarement plus de deux pages, comme autant de vagues sur une dune, dans une suite ininterrompue dont la logique nous échappe mais qui n'est pas sans charmes. Après trente pages de ce régime, intervalle suffisant pour créer l'attente, l'auteur précise son art d'écrire:

Doit-on s'étonner que Les Littératures de l'exiguité soit écrit dans une forme de discontinuité à laquelle Roland Barthes aimait s'adonner? Cette pensée par vignettes plus ou moins brèves marque l'ironie. Entre ces vignettes, dans leurs interstices, la menace sourde et effrayante du silence persiste. Ce n'est pas une manière de déconstruire, comme on le dit souvent: c'est une manière de construire sur le vide. Je ne crois pas que les chapitres, dans leur déploiement agressif,' présomptueux, conviennent aux objectifs que je me suis fixés. Les questions que j'aborde prendront tout leur sens dans la discontinuité. (p. 30)

Sartre disait jadis que le style était une métaphysique. François Paré s'en est souvenu, peut-être, dans cette justification ontologique d'une forme d'écriture. Il est permis, toutefois, de n'être pas convaincu, et de regretter des chapitres et un discours plus composé. Le caractère "agressif et présomptueux" des chapitres ne m'apparaît pas une évidence: du moins, on ne s'est jamais plaint en ma présence d'avoir été ainsi agressé ou snobé. Il y a quelque mauvaise foi à l'affirmer. La position est révélatrice d'un anti-intellectualisme qui est encore plus évident quand l'auteur reviendra, plus loin, sur sa démarche: "Lyotard 
m'avait montré, comme Barthes, la voie de la pensée discontinue, par vignettes, par intuitions, sans que n'interviennent comme une censure intolérable, les structures du discours critique. (p. 58).

Mieux vaut ne pas s'arrêter, je crois, sur ces justifications spécieuses, et d'autant plus que le discours de François Paré est effectivement construit, sinon en chapitres, du moins autour d'un certain nombre de constellations thématiques, et qu'il est un salutaire discours critique, assez structuré. François Paré n'aime pas les chapitres. C'est son droit. Mais j'aurais préféré qu'il n'invoque pas ainsi la "censure intolérable. qu'est un discours critique structuré. On peut choisir une écriture de la discontinuité pour toutes sortes de raisons, entre autres une répugnance ou une incapacité (passagère) à mieux ordonner son propos, sans pour autant accuser la composition discursive de maux imaginaires. On pourrait choisir, aussi, d'insister sur la face positive de l'écriture par intuitions. L'écriture discontinue, en effet, a ses lettres de noblesse. Que l'on songe, par exemple, aux Pensées de Pascal. Son Apologie projetée aurait-elle eu cet effet percutant? Je vois, pour ma part, dans cette composition par vignettes, une écriture tout à fait pertinente pour l'essai, signe que la pensée se cherche, ne s'est pas encore coagulée, et que l'objet de la réflexion ne s'est pas pleinement constitué.

Les idées que François Paré met à l'essai sont d'ailleurs relativement nouvelles et il était singulièrement bien placé pour les articuler. CEuvrant dans le milieu franco-ontarien depuis quelques années, il a été rendu très sensible aux littératures marginales, connexes ${ }^{2}$, minoritaires, embryonnaires:

Il m'est devenu impossible de voir la Littérature - toute la Littérature, voilà la question - autrement que par les yeux inquiets de ces bandes exiguës de culture, ces écritures de l'exiguité, qui me semblent bien souvent constituer aujourd'hui le tranchant de l'écriture mondiale (p. 7 ).

Certes, sans doute pourrait-on citer, parmi les devanciers de François Paré au pays, un Octave Crémazie, dont les commentaires amers sur la marginalité inéluctable de la littérature québécoise ont fait école. Mais le propos de François Paré est autre. En tant que spécialiste de la Renaissance, ce siècle est sa référence constante. Se sont alors constituées institutionnellement les grandes littératures européennes - la française, l'anglaise, l'allemande et, à moindre degré, l'italienne - qui se sont affirmées comme héritières de l'Antiquité gréco-romaine, ont fermement posé les assisses de leur pouvoir implacable, se sont données, en somme, comme souverainement évidentes, pôles obligés de toute réflexion littéraire et de tout discours culturel. 
Sans la moindre trace de mauvaise conscience, elles ont refoulé vers la marge toutes les autres.

C'est dans cet espace des restes que François Paré est allé les chercher, s'attardant davantage aux littératures franco-ontarienne et québécoise, mais embrassant également la suisse ou l'acadienne, la silésienne ou la jamaïcaine, l'islandaise ou la basque, la zaïroise ou l'occitane. Il y a, dans le monde, des milliers de littératures minoritaires, suscitées par l'arbitraire des frontières et les aléas de l'émigration; des centaines de littératures coloniales, legs de l'impérialisme européen; de nombreuses littératures insulaires, à la fois dépendantes et farouchement autonomisantes; et plusieurs petites littératures nationales, dont le degré d'institutionnalisation est variable et facteur des ambitions collectives. La notion d'exiguité, en somme, est élastique. Les écrivains québécois peuvent souffrir de leur marginalité mais celleci n'a aucune commune mesure avec celle des petites cultures qui sont à la limite de l'intelligibilité, puisqu'elles sont hors des grandes langues et dépendantes de la traduction. Ainsi, selon que l'éclairage se portera sur la littérature franco-ontarienne ou la française, la littérature québécoise sera perçue comme hégémonique ou exiguë. Par ailleurs, dans la mesure où elles sont à toutes fins pratiques exclues des programmes d'enseignement des universités occidentales, les littératures du Japon et de l'Inde, pays populeux et puissants, pourraient aussi être perçues comme de petites littératures.

On le voit, la notion est souple. Elle permet à l'auteur des réflexions stimulantes sur des écrivains et des œuvres du Québec, qui sont mises en regard d'une production mondiale. Elle lui permet; davantage, d'aborder sans condescendance des textes francoontariens, que de nombreux Québécois découvriront avec étonnement. Elle lui permet, surtout, de judicieuses réflexions sur la littérature, le discours critique, l'enseignement universitaire, la tyrannie théorique, la prétention à l'universalité de la littérature française et la nécessaire création de "biens symboliques inédits" (p. 157) par une authentique marginalité culturelle.

La réflexion de François Paré s'insère dans une mouvance actuelle qui tend à exacerber les particularismes, à valoriser les singularités, qu'elles soient ethniques ou groupusculaires, et à faire éclater les ensembles sous une forte poussée centrifuge. Crépuscule des nations hégémoniques et déclin de leurs intellectuels? Parcellisation du. pouvoir symbolique et décentralisation radicale du discours? Plusieurs s'en inquiètent ou le déplorent. François Paré, au contraire, s'en réjouit. "La conjoncture est donc à son meilleur" (p. 157), déclare-t-il. 
Les marginaux prennent conscience qu'ils sont «plusieurs "; qu'ils sont, de fait, la majorité. "À elles seules, les grandes littératures ne sont que l'institutionnalisation mémoriale de l'exclusion. (p. 158), exclusion qui a appauvri l'humanité. Or, les exclus clament de plus en plus leur pluralité, affirment l'irréductibilité de leurs différences, manifestent la richesse de leur hétérogénéité, se reconnaissent multiples mais liés.

C'est ainsi sur un ton de manifeste que se termine l'essai discontinu de François Paré. L'essentiel se trouve maintenant dans la marge. Qui devient, du fait même, l'espace fondamental.

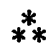

Dans notre histoire culturelle, il n'y a sans doute pas plus marginal que Joseph Guibord, lui qui, même mort, a été repoussé, exclu, refoulé à la marge de la société civile et religieuse de son temps et dont le cadavre, interdit de séjour dans la partie consacrée du cimetière catholique de Montréal, n'a cessé, pendant des années, de se promener dans l'espace fort exigu laissé à la contestation par le pouvoir clérical hégémonique du XIX ${ }^{e}$ siècle québécois.

Or, Joseph Guibord était typographe de son métier. Y aurait-il là quelque symbole à méditer? Sur le destin de l'écrit dans notre société, par exemple? Voici un homme qui a passé sa vie à composer du texte et qui, une fois mort, a été condamné à la marge! Et qui, s'y retrouvant, l'a même réinventée! En effet, lorsque Joseph Guibord, sur ordre du Conseil privé de Londres, fut finalement enterré dans le cimetière catholique, le bouillant Ignace Bourget se retourna aussitôt pour déconsacrer l'espace occupé par la fosse. La marginalité, contre toute logique et toute bonne pratique typographique, se retrouvait en plein centre, et le Canada français réinventait le champ textuel.

Tout cela, le pauvre Joseph Guibord le faisait à son corps défendant, si l'on me passe l'expression. En effet, son cadavre était l'enjeu d'un procès retentissant qui opposait, dans une lutte à finir, les membres de l'Institut canadien, libéraux et anticléricaux, et le plus dogmatique des ultramontains, Ignace Bourget, évêque de Montréal. Avec Le Procès Guibord ou l'Interprétation des restes ${ }^{3}$, Robert Hébert nous convie à une fascinante relecture de cet événement capital, tout à fait central dans notre vie intellectuelle, connu de tous mais réellement étudié par personne. Le même Robert Hébert nous avait offert, il y a quelques années, une remarquable anthologie de textes philosophiques sur l'Amériqué . La même originalité de pensée et la même 
érudition se retrouvent dans cet essai, assez étonnant dans sa facture, mais combien stimulant.

L'étude de Robert Hébert comporte quatre parties: une "Présentation", polémique et percutante, où il identifie les protagonistes du procès Guibord et précise les enjeux de cette affaire retentissante; ensuite les plaidoiries de Joseph Doutre, avocat de madame Guibord et de l'Institut canadien, texte savoureux, savant et baroque, truculent et érudit, rigoureux et pourtant excessif, d'une étonnante et ubuesque modernité (n'appelle-t-il pas Mgr Bourget "le torpédiste interlope" !), et qu'on devrait proposer à nos étudiants plutôt que Les Fiancés de 1812, roman du même, publié en $1844^{\mathrm{s}}$; la troisième partie du livre, à caractère bibliographique, est très courte, mais elle est suivie par le "Carnet du chercheur", qui fait presque la moitié du volume et qui "tient du mémento documentaire et de l'albumm onirique" (p. 8). Dans un indescriptible foisonnement, l'auteur y raconte sa recherche, de 1984 à 1990. Il fait état de ses lectures, de ses réflexions, de ses doutes et de ses découvertes, mais aussi de son rapport de plus en plus passionnel aux acteurs de cette affaire: "Joseph Guibord (inconscient de sa cause) via Joseph Doutre (hyperconscient de ses effets) m'interpellent à partir de leurs fosses " (p. 160). Véritable journal d'un chercheur, ce carnet est du plus puissant effet. On se prend à regretter que les savants et les érudits ne racontent pas plus souvent, sur ce mode fragmenté et discontinu, l'aventure passionnante de leur recherche, narrations qui vaudraient bien, il me semble, si elles ne les surpassaient pas, tellement de récits de découvreurs. Les continents ne sont pas que géographiques.

Mais peut-être ne devrais-je pas opposer ainsi découvertes géographiques et intellectuelles. Robert Hébert, lui, les concilie avec adresse. Dans un passage fascinant de ce Carnet, "Expérience ambulatoire et toponymique "(p. 175-177), l'auteur promène son lecteur à travers les rues de Montréal. D'est en ouest, sous le nom des rues, dans une "sémantique sauvage de la métropole et de la nécropole", il ressuscite tous les acteurs proches ou lointains du procès Guibord et nous révèle leur présence constante dans la vie des Montréalais.

Ce petit exercice de virtuosité historique n'est qu'un indice de l'érudition déployée dans cette étude. L'avocat Joseph Doutre assignait à comparaître l'ensemble de la culture occidentale lors du procès; à son exemple, ce carnet convoque, pour l'instruction du lecteur, nouveau juge saisi de cette affaire, les témoins textuels les plus riches et les plus diversifiés qu'on puisse imaginer. C'est d'ailleurs l'un des sens qu'il faut donner au second titre de cette étude. "L'interprétation des 
restes", c'est, dans un premier temps, l'interprétation des "restes humains" de Joseph Guibord. "La civilisation, la culture sont un art de la sépulture. L'art de mettre les restes humains dans leur continuum historique, signifiant, libérant» (p. 158). En refusant la sépulture chrétienne de ce personnage modeste, le clergé canadien cherche à imposer un sens à sa vie, sens que refuse la famille et les amis libéraux. Mais "l'interprétation des restes" a aussi un autre sens pour l'auteur. Ce conflit local n'est que l'avatar canadien d'une partie immensément plus large, mettant aux prises les grands enjeux philosophiques de l'Occident. Il fait comprendre "trois siècles d'histoire euro-canadienne et québécoise" (p. 8). Pour qui sait lire, il livre les indices, les traces, les empreintes ( $c f$. p. 178) des grands débats philosophiques européens depuis la Renaissance. D'où l'importance de ressusciter les "restes textuels" (p. 174) qui sont évoqués par la plaidoirie de Doutre et qui nous aident à comprendre cette affaire.

Car le procès Guibord est une affaire, tout à fait l'équivalent de l'affaire Dreyfus en France; c'est-à-dire un événement public qui déchaîne les passions, mobilise les groupes sociaux, et cristallise sur un point les grands conflits idéologiques d'une époque. On comprendra que le personnage du typographe est secondaire. Il est prétexte aux deux sens du mot. C'est Joseph Doutre, dans sa "volonté lyrique et érudite de démonstration. (p. 21), qui est le véritable protagoniste de l'affaire et de l'essai de Robert Hébert. Sa plaidoirie est un "texte fondateur" (p. 20), d'une grande puissance analytique et d'une indéniable qualité littéraire, un des grands écrits de la libre pensée. Dix ans avant "La légende du Grand Inquisiteur" de Dostoïevski, "dix ans avant les grandes analyses nietzschéennes des préjugés moraux, des faux rédempteurs, du grégarisme et de l'esprit de soumission" (p. 23), Joseph Doutre démontait les rouages du cléricalisme et de la pensée intolérante. "Cette plaidoirie professionnelle pour la vérité et la tolérance porterait déjà la signature d'un "intellectuel", bien avant l'invention du terme par Émile Zola à l'occasion de l'affaire Dreyfus" (p. 20).

Ce livre est donc, en fait, le tombeau de Joseph Doutre, c'est-àdire son éloge, sa sépulture élogieuse, par un essayiste qui peut se plaindre amèrement que la mémoire oublieuse du Québec n'ait pas su reconnaître auparavant ce penseur peu commun. Tombeau ou exhumation respectueuse, c'est tout comme. Car ce bel essai est aussi une réflexion sur notre ignorance historique et sur le bon usage des morts. Robert Hébert est bien de ces "piocheurs d'antiquités" (p. 82) que souhaitait Joseph Doutre pour nous aider à comprendre notre propre histoire. Celle dont les restes risquent de nous échapper, à moins que nous ne creusions dans les marges de l'histoire officielle. 


\section{***}

Robert Hébert pensait donner, comme second titre à son essai sur l'affaire Guibord: "Éléments pour une herméneutique rouge" (p. 185). Pierre Milot, lui, a effectivement intitulé son livre: Le Paradigme rouge. L'avant-garde politico littéraire des années $70^{6}$. D'un siècle à l'autre, les Rouges sont dans les titres, tout en restant dans la marge sociale. Ils ont plus d'audience, toutefois, un siècle après l'affaire Guibord. Ils ont même de l'autorité, ou du moins parlent avec toutes les marques discursives de celle-ci. Il n'y a plus de $\mathrm{M}^{\mathrm{gr}}$ Bourget pour les refouler vers la brêche et les condamner au silence prudent; l'Église et la droite n'ont plus de hérault.

Cela ne veut pas dire pour autant que la voix des rouges se fait davantage entendre, même s'ils ont maintenant tout loisir de créer des revues à qui mieux mieux. Au contraire. Pierre Milot est le premier à souligner, dès la phrase initiale de son étude sur Socialisme québécois, Stratégie, Chroniques, que les structuro-marxistes des années soixantedix disparaissent très vite de l'horizon culturel québécois. Dès les années quatre-vingt, le champ culturel s'est refermé sur ces revues d'avant-garde, les enterrant sous la plus profonde indifférence. Pierre Milot a-t-il raison, alors, de les rappeler à notre mémoire? Sans doute, encore que la lecture de son étude nous en fasse à l'occasion douter.

Celle-ci est divisée en quatre chapitres précédés d'une introduction théorique et suivis d'une très lacunaire conclusion. L'introduction situe l'entreprise de Pierre Milot face à d'autres, analogues, portant sur des revues plus connues et plus marquantes (La Relève, Parti pris); ensuite, elle précise son cadre analytique (les travaùx de Pierre Bourdieu sur le champ intellectuel), et donne une indication de son parcours:

De la genèse à la chute du paradigme rouge, c'est de l'émergence, de l'institutionnalisation, de la disciplinarisation et de la marginalisation de l'avant-garde politico-littéraire québécoise dans le champ intellectuel et dans le champ universitaire des années 70 que nous avons cherché à rendre compte... (p. 22)

Le premier chapitre décrit "l'avant-garde politico-littéraire parisienne des années soixante, faisant ressortir: a) les rôles joués par Louis Althusser et Roland Barthes dans la redéfinition des études littéraires, sous les assauts d'un nouveau scientisme; b) les efforts du Parti communiste français de se donner une certaine crédibilité intellectuelle en effectuant, par le biais de La Nouvelle Critique, des rapprochements avec les intellectuels d'avant-garde; c) le rôle déterminant de 
Tel quel et de Philippe Sollers dans ce rapprochement et dans l'articulation du marxisme aux théories nouvelles introduites par la réflexion sur les sciences humaines; d) le dérapage causé par Mai 68 et l'émergence du maoïsme dans la cacophonie de gauche; e) la présence constante de Sartre pendant ces années.

Les chapitres suivants seront consacrés à l'analyse des trois revues d'avant-garde québécoise des années soixante-dix qui pratiquent toutes, avec plus ou moins de bonheur et un degré variable de lucidité et d'honnêteté intellectuelle, une forme de parisianisme indigène. Socialisme québécois, d'abord (chapitre II), revue "structuromarxiste", qui prend le relais de Socialisme (dix-neuf numéros de 1964 à 1970) et publie, dans une tonalité sérieuse, universitaire, un grand total de quatre numéros de 1970 à 1974. Ensuite Stratégie (chapitre III), revue à prétention scientifique, qui verse rapidement dans le maoïsme totalitaire, et qui sera sabordée par ses animateurs au profit d'une action politique après qu'ils eurent publié dix-neuf numéros de 1972 à 1977. Finalement Cbroniques (chapitre IV), revue "structuro-marxiste" également, mais moins théorique et universitaire que Socialisme québécois; et qui cherchera à être mensuelle de 1975 à 1978. Les trois revues ont leur identité et leur histoire propres; ou, pour parler comme Pierre Milot, leurs "agents", leurs "stratégies discursives", leurs "indicateurs discursifs", leurs "trajectoires institutionnelles" particuliers; elles se feront même à l'occasion la guerre (Stratégie et Chroniques), fidèles à ce sectarisme qui semble être l'apanage des mouvements de gauche, congénitalement doctrinaires; elles ont pourtant toutes la prétention d'attaquer "l'idéologie dominante " et de travailler à la victoire de la classe ouvrière.

Pierre Milot présente chacune des revues, identifie ses animateurs, précise le "capital littéraire" de ceux-ci (leur réputation, fondée sur la présence ou l'absence d'œuvres) et leur "habitus disciplinaire" (leur formation et leur statut intellectuel), puis rend compte des articles et essais publiés dans les revues. Il les discute les uns à la suite des autres, les citant abondamment, établissant des rapports avec les autres articles de la revue, avec le contexte historique, avec des articles des revues concurrentes, et avec les références françaises canoniques, même quand celles-ci ne sont pas reconnues par les rédacteurs euxmêmes. La démarche tient autant de l'analyse que de la paraphrase. Avec un sérieux méritoire, il discute ce qu'il est permis de considérer comme un ramassis de poncifs, un fatras de lieux communs marxisants, et des doses inhumaines de bons sentiments révolutionnaires (qui, pas plus que les autres, ne font de la bonne littérature...), tous ces gens se réclamant, de plus, de la rigueur méthodologique la plus 
évidente et d'une scientificité impeccable. Scientisme qui, paradoxalement, comme tout fanatisme, est prompt à manier l'invective ou l'excommunication. Les écrivains de La Relève ou de Parti pris, du moins, avaient du talent, tout en ayant infiniment moins de dogmatisme. On en vient donc à admirer Pierre Milot de s'être livré à cet exercice.

Pas trop, tout de même. Car son étude, qu'il appelle "essai" mais qui se drape dans tous les oripeaux de la thèse, y compris la lourdeur de style, le jargon prétendument et prétentieusement rigoureux et les renvois infinis à ses lectures, ne ménage guère son lecteur. Sans doute François Paré pensait-il à de tels écrits quand il parlait du "déploiement agressif, présomptueux • des chapitres discursifs. Ici, rien à redire des chapitres. Mais les phrases pondéreuses, interminables, qui veulent tout dire, y compris résumer tout ce qui a été dit précédemment avant d'avancer d'un pas, taxent là patience du plus bénévolent lecteur. Effet de contamination, sans doute. On n'étudie pas les structuromarxistes impunément.

Le lecteur en vient à regretter que ces rouges des années soixantedix - et leur analyste - ne soient pas retournés lire quelques pages de leurs devanciers. Quelques pages de Joseph Doutre, par exemple, ou encore de Louis-Antoine Dessaulles. Ils y auraient appris qu'on peut être penseur - de gauche, encore! donc marginal - et écrivain.

1. François Paré, Les Littératures de l'exiguïté, Hearst, Le Nordir, 1992, 175p.

2. C'est ainsi, on s'en souviendra, que le volume III de la Pléiade consacré à l'Histoire des littératures abordait les littératures francophones non hexagonales: littératures connexes!

3. Robert Hébert, Le Procès Guibord ou l'interprétation des restes, Montréal, Triptyque, 1992, $196 \mathrm{p}$.

4. Id., L'Amérique française devant l'opinion étrangère (1756-1960). Anthologie, Montréal, l'Hexagone, 1989. Voir mon bref compte rendu dans Voix et Images, $\mathrm{n}^{\circ} 47$.

5. Exemple d'une phrase de Joseph Doutre: «Quant à engager une polémique avec les ignorantins qui s'étouffent dans l'admiration d'eux-mêmes, nous nous réservions le plaisir de les déplumer ici, s'ils trouvaient, dans le barreau, un avocat pour répéter leurs monumentales jocrissades. (p. 41).

6. Pierre Milot, Le Paradigme rouge. L'avant-garde politico-littéraire des années 70 , Candiac, Les Éditions Balzac, coll. *Littératures à l'essai •, 1992, 295 p. 